\title{
ADHERENCIA AL TRATAMIENTO EN LA ESQUIZOFRENIA
}

\section{CARMEN PÉREZ GARCÍA}

Enfermera especialista en Salud Mental y Geriatría.

Residencia SARquavitae Santa Justa (Sevilla).

\section{RESUMEN}

El incumplimiento farmacológico es un problema importante en el ámbito sanitario que se acompaña de un alto coste económico, lo que hace necesario detectar la causa del incumplimiento y qué tipo de usuarios lo llevan a cabo. La observación de esta realidad por parte de enfermería hace que nos replanteemos cuestiones como cuál es el motivo para el abandono/incumplimiento del tratamiento y cómo son nuestras relaciones con los pacientes que no se adhieren a los tratamientos.

Encontrar respuestas para estas preguntas es una cuestión relevante a la hora de contribuir al aumento de la calidad de vida de las personas con esquizofrenia. En las últimas dos décadas se ha investigado sobre el tema y queda evidenciado que enfermería tiene un papel esencial en la reducción de las recaídas y en la mejora del ajuste social, lo que conlleva una mejor adherencia al tratamiento.

Palabras clave: adherencia al tratamiento, esquizofrenia, enfermería.

\section{INTRODUCCIÓN}

La esquizofrenia es una enfermedad mental de las más debilitantes y desconcertantes. La probabilidad de padecer la enfermedad a lo largo de la vida se estima entre el 0,7\% y el 0,9\% de la población en los países europeos ${ }^{1}$. Es un trastorno mental que continúa siendo, a pesar de los avances científicos, insuficientemente conocido.

Correspondencia: C. Pérez.

Correo electrónico: enfermerap8@hotmail.com
La incidencia anual media de la esquizofrenia es de 0,21 por 1000, con escasas diferencias entre los países desarrollados y los del tercer mundo. Sin embargo, no sucede lo mismo con la prevalencia media, que es de 6,3 por 1000 en países desarrollados y de 3,4 por 1000 en los países en desarroIlo. Esta situación puede explicarse en parte por la dificultad de identificar los mismos casos transcurrido un tiempo, y quizá también porque pudiera darse una mayor mortalidad.

El concepto de tratamiento ha ido variando a lo largo de la historia hasta llegar a mediados del siglo 
pasado cuando aparecen los psicofármacos. Hay que destacar que para conseguir la independencia funcional de las personas con enfermedad mental no bastaba con administrar un buen fármaco, sino que se demostró la necesidad de potenciar alternativas de rehabilitación psicosocial².

El incumplimiento en la medicación es la razón primaria de las recidivas, que contribuye al «síndrome de la puerta giratoria» de los pacientes con esquizofrenia ${ }^{3}$.

Sorprende observar que un problema tan importante para la eficacia del tratamiento de la esquizofrenia no haya recibido atención hasta hace relativamente pocos años. Los primeros estudios sobre cumplimiento terapéutico se empezaron a desarrollar 30 años después de la introducción de los neurolépticos. La conferencia de consenso celebrada en Brujas (Bélgica), en 1989, titulada «Directrices para la Prevención de Recidivas de la Esquizofenia con Neurolépticos», fue la primera convención internacional convocada con el fin de diseñar unas directrices para la prevención de las recaídas. En ella ya se señaló el factor de la adherencia como un elemento clave del tratamiento psiquiátrico.

La cronicidad de las patologías mentales, la falta de conciencia de enfermedad o el elevado grado de estigmatización que acompaña a los trastornos mentales son algunos de los factores que agravan el problema de la adherencia en psiquiatría. Existe cierto acuerdo en considerar que la falta de adherencia al tratamiento en las personas con enfermedad mental tiene importantes implicaciones: peor pronóstico, mayor número de hospitalizaciones, mayores tasas de recaídas o recurrencias, mayores tasas de suicidio y peor calidad de vida ${ }^{4-10}$, entre otras.

Según la bibliografía consultada, se puede señalar que los principales factores que intervienen en una mala adherencia al tratamiento son los siguientes ${ }^{11}$ :

- Factores relacionados con el paciente:

- Grado de gravedad de los síntomas
- Insight pobre

- Abuso de alcohol o drogas

- Factores relacionados con la medicación:

- Efectos secundarios

- Dosis subterapéuticas o excesivamente altas

- Factores relacionados con el entorno:

- Soporte o supervisión inadecuados

- Barreras prácticas, como falta de dinero o de transporte

- Factores relacionados con el clínico:

- Alianza terapéutica pobre

Desde el inicio de la desinstitucionalización y con el actual modelo de atención comunitaria, el papel de la familia haciéndose cargo de los pacientes ha sido fundamental, convirtiéndola en el principal recurso para el cuidado y mantenimiento en la comunidad de las personas con enfermedades mentales crónicas, además de reducir el uso a largo plazo de los servicios psiquiátricos. Para la vida familiar, asumir esta tarea ha supuesto consecuencias importantes, si tenemos en cuenta, además, que la desinstitucionalización no ha conllevado, en la mayoría de los casos, una adecuada provisión de servicios y programas de actuación en la comunidad. La familia debe ocuparse de la persona enferma más allá de lo que suponen sus funciones habituales. La función de cuidador añadida a la función familiar habitual es lo que provoca «carga». También hay que señalar que algunos pacientes son una fuente importante de apoyo para su familia, ya que colaboran en las tareas domésticas y proporcionan compañía.

\section{El incumplimiento en la medicación es la razón primaria de las recidivas, que contribuye al «síndrome de la puerta giratoria» de los pacientes con esquizofrenia}


Desde la década de 1950 los familiares de las personas con esquizofrenia comienzan a verse involucrados, en sesiones conjuntas, en el tratamiento de la esquizofrenia. La terapia familiar surge en estos años como «el brazo clínico» de una serie de teorías que postulan el papel etiológico de ciertas características familiares en la aparición de la esquizofrenia. El objetivo general de la terapia familiar basada en estas teorías era la modificación de la dinámica y comunicación familiar, enfatizando los aspectos más negativos de las familiares ${ }^{12}$.

La intervención familiar se ha llegado a considerar una condición imprescindible en el proceso rehabilitador. Varios estudios han demostrado que los pacientes recaen más cuando sus familiares tienen expresiones emocionales altas, es decir, expresiones de crítica, hostilidad o sobreinvolucramiento emocional. Está bien documentado que los pacientes esquizofrénicos que participan en programas de rehabilitación tienen más posibilidades de lograr reincorporarse a la vida social activa y de alcanzar una calidad de vida mejor que aquellos pacientes que no participan en ellos. Aun más, de los pacientes que recibieron estrategias rehabilitadoras, entre el $50 \%$ y el $66 \%$ de ellos funcionaba años más tarde activamente en sus comunidades con pocos síntomas $^{13}$.

En Andalucía se recoge de forma expresa la preocupación de la administración sanitaria por defender no solo los mejores niveles de atención posibles a la población andaluza con problemas de salud mental, sino la intención de favorecer su mejor calidad de vida y su integración social14.

El Plan Integral de Salud Mental incorpora la importancia de satisfacer con adecuados niveles de calidad la respuesta a las diversas necesidades de la población afectada por esquizofrenia, incluyendo las situaciones clínicas de contenido psicosocial, las de integración comunitaria y las de lucha contra el estigma ${ }^{15}$.

Por todo ello, las intervenciones familiares son ahora consideradas una parte fundamental dentro del abordaje psicosocial de la esquizofrenia. La evidencia acumulada en las últimas dos décadas ha servido no solo para poner de manifiesto su eficacia en orden a reducir la tasa de recaída en el paciente y mejorar su ajuste social, sino que también se han producido importantes avances en la delimitación de los constructos, variables y factores implicados en la respuesta familiar ${ }^{16}$.

\section{AVANCES EN EL TRATAMIENTO DE LA ESQUIZOFRENIA}

Los tratamientos para la esquizofrenia han evolucionado, ya que esta enfermedad tiene múltiples formas clínicas que corresponden a distintos sustratos biológicos, y es evidente que un único antipsicótico (o varios que actúen de la misma manera) no puede ser igualmente eficaz en todos los enfermos. Por otra parte, la clínica de la esquizofrenia se modifica a lo largo de la evolución de la enfermedad.

Los avances en el tratamiento de la esquizofrenia se deben a:

1. Una mejor diferenciación de la sintomatología.

2. Un mejor conocimiento de la neurobiología.

3. Los nuevos medicamentos.

4. Los nuevos objetivos.

\section{FÁRMACOS UTILIZADOS EN EL TRATAMIENTO DE LOS TRASTORNOS PSICÓTICOS}

Los fármacos utilizados fundamentalmente en el tratamiento de la esquizofrenia son los Ilamados antipsicóticos, cuyo inicio se remonta a la segunda mitad del siglo Xx, con el descubrimiento de la clorpromazina. Son fármacos que actúan generalmente bloqueando los receptores dopaminérgicos postsinápticos. El descubrimiento de la clozapina supuso un cambio en el perfil de acción de los antipsicóticos, ya que actúa sobre otros sistemas de neurotransmi- 
sión. Debido a esto, se le considera el primero de los Ilamados antipsicóticos atípicos. En la actualidad disponemos de dos grandes grupos de antipsicóticos: Ios antipsicóticos clásicos y los atípicos.

Los antipsicóticos clásicos se caracterizan por ser antagonistas competitivos de los receptores dopaminérgicos, aunque también actúan sobre receptores de otros neurotransmisores (adrenérgicos, muscarínicos, serotoninérgicos, etc.). Además, son eficaces frente a la sintomatología productiva y menos en la defectual, producen muchos efectos indeseables y, en general, no son muy bien aceptados por los pacientes. Son poco eficaces sobre la sintomatología negativa.

Los antipsicóticos atípicos tienen un mecanismo de acción más complejo y selectivo que los clásicos, y como consecuencia tienen un perfil de efectos secundarios más tolerable. Este es uno de los motivos por los que estos psicofármacos se consideran, hoy día, de primera elección en todos los aspectos del tratamiento farmacológico de la esquizofrenia.

Entre las características que tienen los nuevos antipsicóticos podríamos enumerar las siguientes:

- Son eficaces tanto sobre los síntomas positivos como negativos.

- Son eficaces en pacientes resistentes a tratamientos clásicos.

- Son eficaces en las formas agudas y crónicas.

- Producen menos efectos indeseables (afectan menos a la PRL y tienen pocos efectos extrapiramidales y, al parecer, poca incidencia de discinesia tardía).

- Disminuyen el riesgo de recaídas.

- La aceptación por parte del paciente es mayor.

- Disminuyen los días de hospitalización, y la integración sociolaboral y familiar es mejor.

- Son fármacos de primera elección y no únicamente para casos resistentes.

Los antipsicóticos típicos son los más antiguos y se caracterizan por su eficacia en el control de los

\section{El planteamiento frente al problema de mayor o menor adherencia al tratamiento de las personas afectadas de trastorno psicótico se enmarca en el concepto de abordaje multidisciplinar y trabajo en equipo}

síntomas positivos. Por otra parte, los antipsicóticos atípicos se asocian con menos efectos secundarios extrapiramidales y son eficaces tanto en los síntomas positivos como en los negativos. Sin embargo, no están exentos de problemas y son más caros. Uno de los avances en el tratamiento de la esquizofrenia fue el desarrollo de antipsicóticos de administración intramuscular y absorción retardada. La principal ventaja de las presentaciones depot sobre los antipsicóticos orales es que facilitan el cumplimiento del tratamiento prescrito en aquellos pacientes con baja conciencia de enfermedad.

\section{Efectos secundarios}

- Sedación: este efecto adverso puede resultar útil en pacientes agitados, violentos y debe ser evitado en pacientes en los que interese mantener una actividad diaria normal. Los fármacos que lo provocan con mayor intensidad son: clorpromazina, levomepromazina, perfenazina, tioridazina y zuclopentixol. Es necesario advertir al paciente de que no debe conducir ni usar maquinaria peligrosa bajo los efectos de estos fármacos.

- Efectos anticolinérgicos: sequedad de boca, visión borrosa, retención urinaria y estreñimiento, producidos fundamentalmente por el grupo de las fenotiazinas.

- Hipotensión ortostática: ya que estos fármacos pueden bloquear receptores adrenérgicos, sobre todo cuando el fármaco se administra por vía parenteral y en dosis altas. 
- Síndrome neuroléptico maligno: es un efecto poco frecuente pero que puede ser mortal en un 15$20 \%$ de los casos. Se caracteriza por hipertermia, rigidez muscular, alteración de la conciencia y alteraciones respiratorias. Se puede tratar con agonistas dopaminérgicos (bromocriptina) y relajantes musculares.

Según la bibliografía consultada, se puede señalar que los principales factores que intervienen en una mala adherencia al tratamiento son ${ }^{11,17,18}$ :

- Factores relacionados con el paciente. Se señalan como factores que pueden influir de forma negativa: la negación de la enfermedad, la desconfianza en el tratamiento y en los profesionales, la respuesta subjetiva negativa a la medicación, el sentimiento subjetivo desfavorable del paciente sobre la enfermedad o el malestar, las creencias y estigmas culturales, el analfabetismo, las deficiencias sensoriales, la mejoría de su patología, la necesidad de tomar una medicación diariamente, el aislamiento social o el nivel socioeconómico bajo. En cambio, un buen grado de insight y una actitud positiva aumentarán el cumplimiento.

- Factores relacionados con la enfermedad. La evolución, las patologías comórbidas, los aspectos neuropsicológicos y los aspectos psicopatológicos, la gravedad y el subtipo de trastorno influirán en la adherencia al régimen terapéutico.

- Factores relacionados con el tratamiento. Si nos referimos al tratamiento farmacológico, destacan los efectos secundarios desagradables de la medicación (mal sabor, discinesias, etc.) no tratados o no identificados, el tipo de tratamiento, la dosis, la vía de administración y la pauta prescrita.

- Factores relacionados con el entorno del paciente. Destacan:

- La actitud de los familiares y personas significativas ante la enfermedad y su tratamiento. La aparición de un trastorno en un individuo no solo le afecta a él, sino también a las perso- nas con las que convive, y cada nuevo episodio y/o recaída de la enfermedad altera el equilibrio familiar. La familia puede ser tanto un desencadenante como un protector de recaídas. Una elevada emoción expresada, constituida por hostilidad, sobreimplicación emocional y comentarios críticos por parte de la familia se relaciona con un mayor número de recaídas. La intervención familiar mejora el clima familiar, el funcionamiento social, la discriminación y reduce las recaídas.

- La relación paciente-profesional. La existencia de una relación terapéutica, con un buen grado de insight es un factor muy importante para ayudar al paciente en todo su proceso terapéutico.

Existen diferentes intervenciones, desde perspectivas multidisciplinares, para incidir favorablemente sobre los diferentes y múltiples factores que se relacionan con la no adherencia al tratamiento ${ }^{19}$. En ellas participan los profesionales que, de forma multidisciplinar, forman parte del tratamiento integral en las personas diagnosticadas de esquizofrenia: psiquiatra, enfermera especialista en Salud Mental, psicólogo, trabajador social y terapeuta ocupacional. No podemos olvidar la ayuda incansable en el tratamiento de la esquizofrenia de los familiares, que junto con los profesionales conforman uno de los pilares fundamentales para conseguir una adecuada adherencia al tratamiento. Estas intervenciones podríamos desglosarlas en: a) intervenciones simplificadoras del tratamiento, $b$ ) intervenciones informativas/ educativas, $c$ ) intervenciones de apoyo familiar/social, d) dinámica de grupos, e) de refuerzo conductual y f) combinación de varias intervenciones.

\section{Papel de la enfermera en la adherencia al tratamiento}

El planteamiento principal que enfermería puede formularse frente al problema de mayor o menor adherencia al tratamiento de las personas afectadas de trastorno psicótico se enmarca en el concepto de 


\section{El papel de enfermería es muy importante, tanto en el abordaje integral como en la toma de la medicación}

abordaje multidisciplinar y trabajo en equipo. Desde los diferentes dispositivos que conforman una unidad de gestión de salud mental, la aportación de enfermería va encaminada a este abordaje integral de la persona que tenemos que tratar, centrándonos sobre todo en ese principal objetivo y el marco común donde se deben encontrar todas las acciones de todos los profesionales, que no es otro que un planteamiento multidisciplinar unidireccional, donde cada profesional "aporta su grano de arena» en el objetivo común: cuidar y mejorar/ayudar a la persona que necesita atención.

Un segundo planteamiento que es necesario señalar es la importancia de las contribuciones individuales que desde cada ámbito profesional se pueden realizar en el abordaje global. En este sentido, la intervención de enfermería tiene una característica muy específica que puede resultar relevante en el resultado final del abordaje. Nos referimos a la proximidad en la atención, entendida tanto desde la frecuencia de contactos como desde la percepción que el paciente tiene del profesional de enfermería. En la práctica son diversas las ocasiones en las cuales el paciente acude a la enfermera para consultar dudas, comentar sensaciones, expresar problemas, etc. También, en otras ocasiones, acude a la enfermera para compartir buenas noticias, alegrías, sensaciones agradables y proyectos de futuro. Esta realidad debe ser tenida en cuenta en todo programa global de intervención.

\section{DISCUSIÓN}

El planteamiento principal que enfermería puede formularse frente al problema de mayor o menor adhe- rencia al tratamiento de las personas afectadas de trastorno psicótico está enmarcado en el concepto de abordaje multidisciplinar y trabajo en equipo. Lo importante es poder trabajar desde una perspectiva multidisciplinar, donde cada profesional que atiende al paciente es una parte importante del complejo puzle que forma el tratamiento integral del paciente con esquizofrenia.

Un segundo planteamiento muy importante es la proximidad en la atención, tanto desde la frecuencia de contactos como desde la percepción que el paciente tiene del profesional de enfermería. Si nos posicionamos en el abordaje de la enfermera encontramos como principales diagnósticos de enfermería según la clasificación NANDA:

1. Incumplimiento del tratamiento, definido como: conducta de una persona... que no coincide con un plan terapéutico o de promoción de la salud acordado entre la persona, etc., y un profesional del cuidado de la salud, etc. Pueden respetarlo total o parcialmente o no cumplirlo en absoluto, lo que puede conducir a resultados clínicos efectivos, parcialmente efectivos o inefectivos ${ }^{20}$.

2. Manejo inefectivo del régimen terapéutico, definido como: patrón de regulación e integración en la vida diaria de un programa de tratamiento de la enfermedad y de sus secuelas que resulta insatisfactorio para alcanzar objetivos específicos de salud $^{20}$

Existe un acuerdo general en aceptar la necesidad de abordajes integrales (biopsicosociales), pero también parece que el determinante o eje directivo del abordaje de la persona afectada de esquizofrenia sea el cumplimiento de la pauta farmacológica. El papel de enfermería es muy importante, tanto en el abordaje integral como en la toma de la medicación.

Evidentemente interviene de forma muy directa en el seguimiento y supervisión del tratamiento farmacológico, especialmente cuando se trata de la administración de inyectables. Administrar el trata- 
miento no es solamente la técnica, sino también todas las intervenciones encaminadas a poder ayudar al paciente a conseguir un grado mayor de autonomía, sin dejar de lado las dificultades que pueden presentar para él la integración del régimen terapéutico en su vida y en su integración en la comunidad.

\section{BIBLIOGRAFÍA}

1. Otaduy Zubia M. Psicoeducación a familias de pacientes con esquizofrenia en una unidad de hospitalización breve. Metas Enferm. 2007;10(6):50-3.

2. Florit Robles A. La rehabilitación psicosocial de pacientes con esquizofrenia crónica. Apuntes de Psicología. 2006;24(1-3):223-44. Disponible en: http:// www.cop.es/delegaci/andocci/files/contenidos/VOL24_ 1_10.pdf

3. Kissling W. Compliance, quality assurance and standards for relapse prevention in schizophrenia. Acta Psychiatr Scand. 1994;89(suppl 382):16-24.

4. Weiden PJ, Olfson M. Cost of relapse in schizophrenia. Schizophr Bull. 1995;21:419-29.

5. Ayuso-Gutiérrez JL, del Río J. Factors influencing relapse in the long-term course of schizophrenia. Schizophr Res. 1997;28:199-206.

6. Hawton K, Suttton L, Haw C, Sinclair J, Deeks JJ. Schizophrenia and suicide: systematic review of risk factors. Br J Psychiatry. 2005;187:9-20.

7. Linares Pellicer MC, Palau Muñoz C, Albiach C, Santos $P$. Predictive variables of adherence to treatment in alcoholics. Actas Esp Psiquiatr. 2002;30:370-5.

8. Knapp M, King D, Pugner K. Nonadherence to antipsychotic medication regimens: associations with resource use and costs. Br J Psychiatry. 2004;184:509-16.

9. Haywood TW, Kravitz HM, Grossman LS. Predicting the "revolving door» phenomenon among patients with schizophrenic, schizoaffective and affective disorders. Am J Psychiatry. 1995;152:856-61.

10. Fleischhacker WW, Oehl MA, Hummer M. Factors influencing compliance in schizophrenia patients. J Clin Psychiatry. 2003;64(Suppl. 16):10-3.
11. Vicens E, Tort V. El incumplimiento de la medicación y las implicaciones clínicas a largo plazo en la esquizofrenia en el ámbito penitenciario. Rev Esp Scand Penit. 2005;7:68-76.

12. Fenton WS, Blyler CR, Heinssen RK. Determinants of medication compliance in schizophrenia: empirical and clinical findings. Schizophr Bull. 1997;23(4):63751.

13. Toruiño González R, Inglott Domínguez R, Baena Ruiz E, Fernández Fernández J. Guía de Intervención Familiar en la Esquizofrenia. 2. ${ }^{a}$ ed. Barcelona: Glosa; 2007.

14. Díaz Martínez LR, Ortega Soto H, Leaños Guerrero C, Rodríguez Verdugo MS, Rascón Gasca ML, Valencia Collazos M, et al. La rehabilitación integral del paciente esquizofrénico en México: el modelo del Instituto Nacional de Psiquiatría Ramón de la Fuente, Grupo III. Salud Mental [Internet]. 2005;28(6):9-19. Disponible en: http://www.inprf-cd.org.mx/pdf/sm2806/sm280 609.pdf

15. Laviana Cuetos M. La atención a las personas con esquizofrenia y otros trastornos mentales graves desde los servicios públicos: una atención integral e integrada en un modelo comunitario. Apuntes de Psicología [Internet]. 2006;24(1-3):345-73. Disponible en: http:// www.cop.es/delegaci/andocci/files/contenidos/VOL24_ 1_16.pdf

16. Junta de Andalucía. II Plan Integral de Salud Mental 2008-2012. Sevilla: Junta de Andalucía, Consejería de Salud; 2008.

17. Gonzalez J, Cuixart I, Manteca H, Carbonell M, Armengol J, Azcon MA. La baja adherencia al tratamiento antipsicótico: actuación de enfermería. Interpsiquis [Internet]. 2004. [Consultado el 20 de febrero de 2006]. Disponible en: http://www.psiquiatria.com.

18. García I, Sánchez El, Sanz M, Gutiérrez M, González de Chavez M. Factores relacionados con la adherencia al tratamiento en pacientes esquizofrénicos. Actas Esp Psiquiatr. 1999;27(4):211-6.

19. Orueta R. Estrategias para mejorar la adherencia terapéutica en patologías crónicas. Información Terapéutica del sistema Nacional de Salud. Madrid: Ministerio de Sanidad y Consumo, 2005.

20. North American Nursing Diagnosis Association (NANDA). Diagnósticos enfermeros: definiciones y clasificación 2003-2004. Madrid: Harcourt; 2004. 